\title{
CORRIGENDUM, VOLUME 82
}

Abraham Zaks, Half factorial domains pp. 721-723.

In $\S 1$ the second paragraph is to read:

A Dedekind domain is called special, if whenever a nonprincipal prime $P$ equals (in $C(R)$ ) a product of primes $Q_{1} \cdots Q_{t}$, it already equals one of the $Q$ 's (in $C(R)$ ).

In $\S 2$ the Proposition 2 is to read:

Proposition 2. Suppose that in the Dedekind domain $R$, for every prime ideal $M$ there exists a prime ideal $N$ so the $M N$ is a principal ideal. Then $R$ is an HFD iff $R$ is a special Dedekind domain whose class group is a direct sum of a free group and a 2elementary group.

AMS (MOS) subject classifications (1970). Primary 10A25, 13F99; Secondary 12A45, $13 \mathrm{D} 15$.

BULLETIN OF THE

AMERICAN MATHEMATICAL SOCIETY

Volume 82, Number 6, November 1976

\section{CORRECTION, VOLUME 82}

Robert C. Reilly, Applications of the integral of an invariant of the Hessian, pp. 579-580.

Equation (1) should read:

$$
\begin{aligned}
\int_{M} 2 S_{2}(f) \Omega= & \int_{N}\left\{\left(\Delta z-u K_{1}\right) u-\langle\nabla z, \nabla u\rangle-\operatorname{II}(\nabla z, \nabla z)\right\} \Psi \\
& +\int_{M} \operatorname{Ric}(\operatorname{grad} f, \operatorname{grad} f) \Omega .
\end{aligned}
$$

Equation (2) should read

$$
1 \geqslant \epsilon\left(\frac{2 \alpha}{1+\epsilon}\right)^{1 / 2} \int_{t_{1}}^{T}\left(\frac{V\left(t_{1}\right)}{V(t)}\right)^{\epsilon} d t
$$

AMS (MOS) subject classifications (1970). Primary 53C20, $53 C 40$. 